\title{
On the ergodic properties of piecewise linear perturbations of the twist map
}

\author{
M. WOJTKOWSKI \\ Departement of Mathematics, Facultés Universitaires de Namur, \\ Rempart de la Vierge 8, B-5000 Namur, Belgium
}

(Received 10 January 1982)

Dedicated to the memory of V. M. Alexeyev

Abstract. It is proved that for a sequence of arbitrarily small piecewise linear perturbations of the twist map, there is a domain with stochastic behaviour (almost hyperbolicity). The measure of this domain has the asymptotics

$$
\frac{1}{16} A \ln \frac{1}{A}(1+o(1)), \quad A \rightarrow 0
$$

where $\boldsymbol{A}$ is the magnitude of the perturbation.

\section{Introduction}

It is not known whether a small area-preserving perturbation of the twist map has an ergodic component of positive measure. The simplest example of such a perturbation is the so-called standard mapping [1]

$$
(I, \theta) \mapsto\left(I+\frac{K}{2 \pi} \sin 2 \pi \theta, \theta+I+\frac{K}{2 \pi} \sin 2 \pi \theta\right), \quad(I, \theta) \bmod 1, K \in \mathbb{R} .
$$

In the present paper, we consider a family of transformations defined by the formula above in which the sinus is replaced by a piecewise linear function with zero mean value. We obtain a two-parameter family of piecewise linear transformations of the torus. For the zero value of the parameters, we get the twist mapping. We prove that, for a sequence of parameters converging to zero, there is an invariant domain in which the transformation has strong mixing properties. In particular, it consists of ergodic components of positive measure. We conjecture that actually in all cases under consideration, there is only one ergodic component and so, as a consequence, the transformation is Bernoulli in the domain. This domain is a small neighbourhood of the transversally intersecting stable and unstable separatrices of a hyperbolic fixed point. Its area has the asymptotics

$$
\frac{1}{16} A \ln \frac{1}{A}(1+o(1))
$$

where $A \searrow 0$ is the parameter.

In the appendix, we prove that, at least for some values of the parameters, the transformation possesses an infinite number of elliptic periodic points with different periods. 
The present paper is the continuation of the paper [2] in which only large perturbations were considered. The reader is advised to consult [2] for more motivations and references.

\section{Description of the transformation}

Let $\Pi^{2}$ be a two-dimensional torus with coordinates $\left(\phi_{1}, \phi_{2}\right) \bmod 1$. Let us consider the twist mapping $F_{1}: \mathbf{T}^{2} \rightarrow \mathbf{T}^{2}$,

$$
F_{1}\left(\phi_{1}, \phi_{2}\right)=\left(\phi_{1}+\phi_{2}, \phi_{2}\right)
$$

and the mapping $F_{2}: \mathbf{T}^{2} \rightarrow \mathbf{T}^{2}$ defined by

$$
F_{2}\left(\phi_{1}, \phi_{2}\right)=\left(\phi_{1}, \phi_{2}+f\left(\phi_{1}\right)\right)
$$

where $f$ is a real function, periodic with period 1 .

Both $F_{1}$ and $F_{2}$ preserve the Lebesgue measure $d \phi_{1} d \phi_{2}$. We shall study transformations of the form

$$
F=F_{1} \circ F_{2}, \quad F\left(\phi_{1}, \phi_{2}\right)=\left(\phi_{1}+\phi_{2}+f\left(\phi_{1}\right), \phi_{2}+f\left(\phi_{1}\right)\right) .
$$

This transformation with

$$
f(t)=\frac{K}{2 \pi} \sin 2 \pi t
$$

is called the standard mapping and appears quite frequently in physical applications [1].

Such transformations can be viewed as perturbations of the twist mapping $F_{1}$ if only $f$ is in some sense small. Our aim is to study ergodic properties of such transformations with respect to the Lebesgue measure.

It is useful to introduce the following change of variables

$$
x_{1}=\phi_{1}-\phi_{2}, \quad x_{2}=\phi_{1} \text {. }
$$

We denote our transformation in $x$-coordinates by $T$. We have

$$
T\left(x_{1}, x_{2}\right)=\left(x_{2},-x_{1}+2 x_{2}+f\left(x_{2}\right)\right) .
$$

Our transformation possesses important symmetry properties. Let

$$
S\left(x_{1}, x_{2}\right)=\left(x_{2}, x_{1}\right) \text {. }
$$

$T$ is $S$-reversible, i.e.

$$
S \circ T \circ S=T^{-1} .
$$

Further, suppose that $f$ is odd with respect to one of its zeros, i.e. $f(z)=0$,

$$
f(2 z-t)=-f(t) \text {. }
$$

Consider the symmetry $P_{z}$,

$$
P_{z}\left(x_{1}, x_{2}\right)=\left(2 z-x_{2}, 2 z-x_{1}\right) .
$$

$T$ is also $P_{z}$-reversible

$$
P_{z} \circ T \circ P_{z}=T^{-1} .
$$

Combining (1) and (2), we obtain

$$
\left(S P_{z}\right) \circ T \circ\left(S P_{z}\right)^{-1}=T
$$

and $S P_{z}$ is the central symmetry with respect to the point $(z, z)$. 
We specify $f$ to be piecewise linear with two pieces in the domain of one period and such that

$$
\int_{0}^{1} f(t) d t=0 .
$$

The last equality is the necessary condition for transformation $F$ to have an invariant curve of the form $\phi_{2}=h\left(\phi_{1}\right)$, where $h$ is a continuous periodic function. Indeed, consider the transformation $F$ on the cylinder $\left(\phi_{1}, \phi_{2}\right), \phi_{1} \bmod 1$. The curve

$$
\phi_{2}=h\left(\phi_{1}\right)+N, \quad n \in \mathbb{Z},
$$

does not intersect the curve

$$
\phi_{2}=-f\left(\phi_{1}\right) \text { if } N \text { is sufficiently large. }
$$

The areas of the annulus between the curves and its image coincide. But the first curve is invariant and the image of the second has the form $\phi_{2}=0$. We conclude that

$$
\int_{0}^{1} f(t) d t=0 .
$$

Clearly, the shift of the function $f$ is equivalent to the shift of coordinates. So we are left with a two-parameter family of periodic functions with period 1

$$
f_{A, B}(t)=\left\{\begin{array}{rr}
-A t-c / 4 & -2 a \leq t \leq 0, \\
B t-c / 4 & 0 \leq t \leq 2 b,
\end{array}\right.
$$

where $c$ is the harmonic mean of $A$ and $B$,

$$
c=2 A B /(A+B), \quad a=c / 4 A, \quad b=c / 4 B,
$$

$A>0, B>0$. We see that $f$ is odd with respect to its zeros: $-a$ and $b$. The symmetry transformations $P_{-a}$ and $P_{b}$ coincide when considered on the torus.

It was proved in [2] that, for $A=B, T$ has strong mixing properties in the whole torus if $A \geq 4$ and in some invariant domains for a sequence of parameters $A$ from the interval $[1,4]$. So $A$ was quite far from zero. To obtain analogous results for arbitrarily small $f$, we shall need two parameters (we shall consider 'co-dimension 2 phenomenon').

We take the following fundamental domain in the coordinate plane $\left(x_{1}, x_{2}\right)$ :

$$
\left\{\left(x_{1}, x_{2}\right) \mid-2 a \leq x_{i} \leq 2 b, i=1,2\right\} \text {. }
$$

Further let

$$
\begin{array}{cl}
\mathscr{B}_{+}=\left\{\left(x_{1}, x_{2}\right) \mid 0 \leq x_{2} \leq 2 b\right\}, & \mathscr{B}_{-}=\left\{\left(x_{1}, x_{2}\right) \mid-2 a \leq x_{2} \leq 0\right\}, \\
\mathscr{G}_{+}=\left\{\left(x_{1}, x_{2}\right) \mid 0 \leq x_{1} \leq 2 b\right\}, & \mathscr{G}_{-}=\left\{\left(x_{1}, x_{2}\right) \mid-2 a \leq x_{1} \leq 0\right\} .
\end{array}
$$

We have that $T$ is linear in $\mathscr{B}_{+}$and in $\mathscr{B}_{-}$and $T\left(\mathscr{B}_{ \pm}\right)=\mathscr{G}_{ \pm}$. In $\mathscr{B}_{+}$, there is a fixed point $(b, b)$ and in $\mathscr{B}_{-}$a fixed point $(-a,-a)$. The matrix of $T(D T)$ in $\mathscr{B}_{+}$is

$$
M_{+}=\left(\begin{array}{cc}
0 & 1 \\
-1 & 2+B
\end{array}\right)
$$

and in $\mathscr{B}_{-}$,

$$
M_{-}=\left(\begin{array}{cc}
0 & 1 \\
-1 & 2-A
\end{array}\right)
$$


The first matrix is hyperbolic when $B>0$ i.e. it has real eigenvalues outside the unit circle. We assume that $0<A<2$, so that the second matrix is elliptic i.e. it has complex-conjugated eigenvalues on the unit circle.

\section{Invariant curves}

We are going to show that, for arbitrarily small special values of $A$ and $B$, there is a domain in the torus in which the transformation is almost hyperbolic. This domain is bounded by piecewise linear invariant curves. We shall see that such curves exist for special values of $A$ and $B$.

We are interested in invariant curves in some sense close to the diagonal $x_{1}=x_{2}$. The orbit of any point, unless it stays all the time in $\mathscr{B}_{+}$or $\mathscr{B}_{-}$, has to pass through $\mathscr{B}_{-} \cap \mathscr{G}_{+}$. These remarks justify the following assumption. Let $\delta$ be a piecewise linear invariant curve such that its intersection with $\mathscr{B}_{-} \cap \mathscr{G}_{+}$is a segment with ends

$$
p=(x, 0), \quad 0 \leq x \leq 2 b,
$$

and

$$
T p=(0,-x-c / 4) \text {. }
$$

In view of the reversibility properties of $T$, we have that $S \delta$ and $P_{-a} \delta$ are also invariant curves. But the curve $\delta$ has to intersect the axis of the symmetry $P_{-a}$. Hence $\delta$ and $P_{-1} \delta$ intersect. It is not difficult to see t'.at either $\delta$ is $P_{-a}$-symmetric (i.e. $P_{-a} \delta=\delta$ ) or $\delta \cup P_{-a} \delta$ is the sum of two $P_{-a}$-symmetric invariant curves (the last possibility will be actually ruled out by the subsequent analysis). So we assume that $\delta$ is $P_{-a}$-symmetric.

Consequently, our symmetric invariant curve $\delta$ must contain the segment with ends $P_{-a} p$ and $P_{-a} T p$. Moreover, some positive iterate $T^{K} p, K \geq 2$, belongs to the segment and the whole orbit $\left\{T^{i} p\right\}, 0 \leq i \leq K$, lies on a $P_{-a}$-symmetric ellipse with the centre at $(-a,-a)$. We conclude that there exists $K \geq 2$ such that

$$
T^{K} p=P_{-a p} .
$$

LEMMA 1. For any $0<A<2, B>0$, there exists a finite sequence $u_{2}>u_{3}>\cdots>u_{n} \geq$ 0 such that, for $p_{K}=\left(u_{K}, 0\right)$, we have

$$
T^{K} p_{K}=P_{-a} p_{K} \text { and } T^{i} p_{K} \in \mathscr{B}_{-} \text {for } 0 \leq i \leq K-1
$$

and there are no other points $p=(x, 0), x \geq 0$, with this property. Moreover,

$$
u_{K}=\frac{1}{8} c(\operatorname{ctg}(K-1) \alpha / 2 \operatorname{ctg} \alpha / 2-1), \quad k=2, \ldots, n
$$

where

$$
\cos \alpha=1-A / 2, \quad 0<\alpha<\pi / 2
$$

and

$$
n=\left[\frac{\pi}{\alpha}\right]
$$

$([\cdot]$ stands for integer part $)$.

Proof. The lemma is obtained by straightforward computations once we observe that we have to apply only iterations of one linear map with the matrix $M_{-}$. We 
get the specific formula for $u_{K}$ by expressing $M_{-}^{K}$ in the following form

$$
M_{-}^{K}=\left(\begin{array}{cc}
\frac{-\sin (K-1) \alpha}{\sin \alpha} & \frac{\sin K \alpha}{\sin \alpha} \\
\frac{-\sin K \alpha}{\sin \alpha} & \frac{\sin (K+1) \alpha}{\sin \alpha}
\end{array}\right)
$$

where $\alpha \in(0, \pi / 2)$ is the angle of rotation of the matrix $M_{-}$, i.e.

$$
\cos \alpha=1-A / 2 \text {. }
$$

We get immediately

$$
u_{K}=a\left(\frac{\sin \alpha+\sin K \alpha}{\sin (K-1) \alpha}-1\right) .
$$

Using trigonometric identities, we obtain the formula above.

Further we assume that our curve $\delta$ is either $P_{b}$-symmetric or $S$-symmetric (the justification of this assumption is the same as above). We conclude in the analogous manner that there is $L \geq 0$ such that

$$
T^{-L} p=P_{b} p \quad \text { in case of } P_{b} \text {-symmetry }
$$

and

$$
T^{-L} p=S p \quad \text { in case of } S \text {-symmetry. }
$$

We use the fact that, for linear hyperbolic transformations, the orbits lie on branches of hyperbolae, in our case either $\boldsymbol{P}_{b}$-symmetric or $S$-symmetric.

LEMMA 2. For any $0<A<2, B>0$, there exist two infinite sequences

$$
2 b=v_{1}>v_{2}>\cdots>v_{\infty} \text { and } 0=v_{-1}<v_{-2}<\cdots>v_{\infty}
$$

such that, for $p_{j}=\left(v_{j}, 0\right)$, we have $T^{-i} p_{j} \in \mathscr{G}_{+}$for $0 \leq i \leq|j|-1$,

$$
T^{j+1} p_{j}=S p_{j}, \quad j=-1,-2, \ldots
$$

and

$$
T^{-j+1} p_{j}=P_{b} p_{j}, \quad j=1,2, \ldots
$$

and there are no other points $p=(x, 0), 0 \leq x \leq 2 b$ with this property. Moreover,

$$
\begin{aligned}
v_{-L} & =\frac{1}{8} c(\operatorname{th} L \beta / 2 \operatorname{cth} \beta / 2-1), \\
v_{L} & =\frac{1}{8} c(\operatorname{cth} L \beta / 2 \operatorname{cth} \beta / 2-1), \quad L=1,2, \ldots, \\
v_{\infty} & =\frac{1}{8} c(\operatorname{cth} \beta / 2-1)
\end{aligned}
$$

where

$$
\operatorname{ch} \beta=1+B / 2, \quad \beta>0 .
$$

Proof. The existence of the two sequences is clear once we observe that we apply only iterations of one linear map with the hyperbolic matrix $M_{+}$. To obtain a convenient expression for $\boldsymbol{M}_{+}^{K}$, we go over to the coordinates in which $M_{+}$is given by the matrix of a hyperbolic rotation

$$
\left(\begin{array}{rr}
\operatorname{ch} \beta & -\operatorname{sh} \beta \\
-\operatorname{sh} \beta & \operatorname{ch} \beta
\end{array}\right)
$$


where $\operatorname{ch} \beta=1+B / 2$. Such change of coordinates is defined by the matrix

$$
\left(\begin{array}{rr}
1 & -\operatorname{ch} \beta \\
0 & \operatorname{sh} \beta
\end{array}\right)
$$

In these coordinates, the matrix $M_{+}^{K}$ has the form

$$
\left(\begin{array}{rr}
\operatorname{ch} K \beta & -\operatorname{sh} K \beta \\
-\operatorname{sh} K \beta & \operatorname{ch} K \beta
\end{array}\right)
$$

Returning to the original coordinates, we obtain

$$
M_{+}^{K}=\left(\begin{array}{cc}
\frac{-\operatorname{sh}(K-1) \beta}{\operatorname{sh} \beta} & \frac{\operatorname{sh} K \beta}{\operatorname{sh} \beta} \\
\frac{-\operatorname{sh} K \beta}{\operatorname{sh} \beta} & \frac{\operatorname{sh}(K+1) \beta}{\operatorname{sh} \beta}
\end{array}\right) .
$$

Now we get immediately the following formulae

$$
\begin{gathered}
v_{-L}=b\left(1-\frac{\operatorname{sh}(L-1) \beta+\operatorname{sh} \beta}{\operatorname{sh} L \beta}\right), \\
v_{L}=b\left(1-\frac{\operatorname{sh}(L-1) \beta-\operatorname{sh} \beta}{\operatorname{sh} L \beta}\right) .
\end{gathered}
$$

Using the identities for hyperbolic functions, we obtain the formulae above.

In the following, we will use $\alpha$ and $\beta, 0<\alpha<\pi / 2, \beta>0$, as alternative parameters of our transformation $T$, instead of $A$ and $B$. For small $\alpha$ and $\beta$, the connection between the parameters is given by

$$
A=\alpha^{2}+0\left(\alpha^{4}\right), \quad B=\beta^{2}+0\left(\beta^{4}\right) .
$$

If our transformation $T$ has an invariant curve $\delta$ with properties specified above, then there must be a coincidence between some $u_{K}, K \geq 2$, and $v_{L}$ or $v_{-L}, L \geq 1$, and vice-versa; if there is such a coincidence, then $T$ has an invariant curve. We will call such an invariant curve a curve of type $(K, \pm L)$. A curve of type $(K, \infty)$ is a separatrix: the curve consisting of the stable and unstable 'manifolds' of the hyperbolic fixed point $(b, b)$.

LemMA 3. Let us fix $K, K \geq 2$, and $L, L=1,2, \ldots, \infty$. For every $\beta>0$, there is a unique

$$
\alpha=F_{K, \pm L}(\beta), \quad \alpha \in(0, \pi / K],
$$

such that the corresponding transformation $T$ possesses the invariant curve of type $(K, \pm L)$. Moreover, $F_{K, \pm L}$ is an increasing differentiable function,

$$
\begin{gathered}
\lim _{\beta \rightarrow+\infty} F_{K, \pm L}(\beta)=\frac{\pi}{K}, \\
F_{K,+L}(0)=0, \quad F_{K,-L}(0)=\alpha_{0}
\end{gathered}
$$

where

$$
\operatorname{tg}(K-1) \alpha_{0} / 2 \operatorname{tg} \alpha_{0} / 2=1 / L
$$


Proof. $T$ possesses an invariant curve of type $(K,+L)$ when $u_{K}=v_{+L}$. This equation is equivalent to

$$
\operatorname{tg}(K-1) \alpha / 2 \operatorname{tg} \alpha / 2=\operatorname{th} L \beta / 2 \text { th } \beta / 2 \text {. }
$$

We see that the left-hand side is an increasing function from $[0, \pi / K]$ to $[0,1]$ and the right-hand side is an increasing function from $[0,+\infty)$ to $[0,1)$. Hence, we obtain the increasing differentiable function

$$
F_{K,+L}:[0,+\infty) \rightarrow[0, \pi / K)
$$

such that $(6)$ is equivalent to

$$
\alpha=F_{K,+L}(\beta) .
$$

Analogously, the equation $u_{K}=v_{-L}$ can be written as

$$
\operatorname{tg}(K-1) \alpha / 2 \operatorname{tg} \alpha / 2=\operatorname{cth} L \beta / 2 \operatorname{th} \beta / 2 .
$$

Now, the right-hand side is an increasing function from $[0,+\infty)$ to $[1 / L, 1)$. So we obtain the increasing function $F_{K,-L}:[0,+\infty) \rightarrow\left[\alpha_{0}, \pi / K\right)$ where

$$
\operatorname{tg}(K-1) \alpha_{0} / 2 \operatorname{tg} \alpha_{0} / 2=1 / L .
$$

It follows from lemma 3 that generally in one-parameter families of transformations $T$, we have piecewise linear invariant curves for a sequence of parameters. For instance, if we consider the one-parameter family with $A=B$, we obtain that, for every $K \geq 2$ and $L \geq 1$, there is a transformation with an invariant curve of type $(K,-L)$ and, if $L \geq K$, then there is also a transformation with an invariant curve of type $(K,+L)$. We obtain this corollary by inspecting the intersection of the graph of the function $F_{K, \pm L}$ with the curve $A=B$ in the $(\alpha, \beta)$ place. The sufficient condition for the intersection with the graph of the function $F_{K,+L}$ is in view of (5)

$$
\left.\frac{d F_{K,+L}}{d \beta}\right|_{\beta=0}>1 .
$$

Expanding (6) in powers of $\alpha$ and $\beta$, we obtain

$$
\frac{1}{4}(K-1) \alpha^{2}+o\left(\alpha^{4}\right)=\frac{1}{4} L \beta^{2}+o\left(\beta^{4}\right),
$$

so that

$$
\left.\frac{d F_{K,+L}}{d \beta}\right|_{\beta=0}=\sqrt{L /(K-1)}
$$

On the other hand, the presence of two piecewise linear invariant curves in a one-parameter family of transformations $T$ is a rare phenomenon. In this case we need essentially two parameters.

Proposition 1. For every integer $K_{1}, K_{2}, L_{1}, L_{2}, K_{2}>K_{1} \geq 2, L_{1} \geq 1, L_{2} \geq 1$, there exist $A$ and $B, 0<A<2, B>0$, such that the corresponding transformation $T$ has invariant curves of type $\left(K_{1},+L_{1}\right)$ and of type $\left(K_{2},-L_{2}\right)$.

Proof. We have to prove that the graphs of $F_{K_{1},+L_{1}}$ and $F_{K_{2},-L_{2}}$ intersect in the $(\alpha, \beta)$-plane which is obvious from figure 1. 


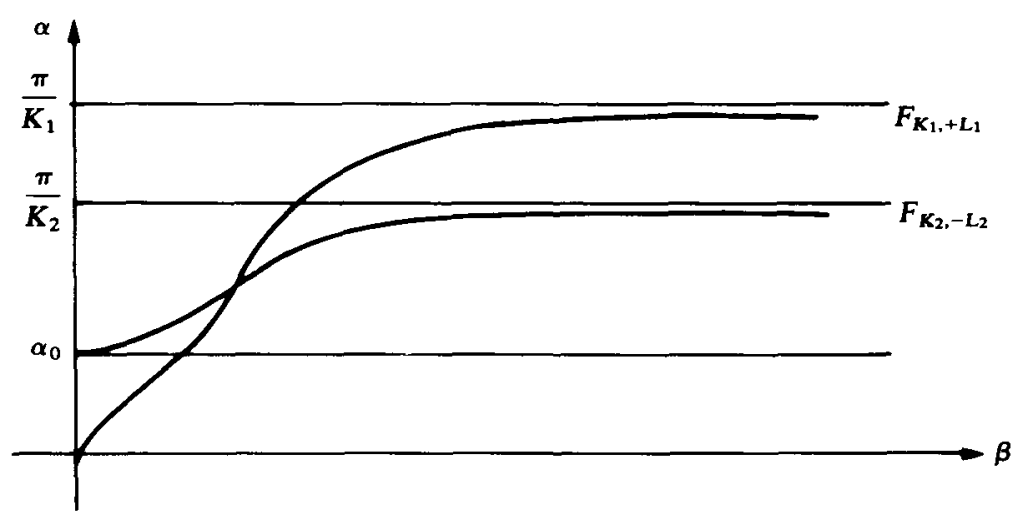

Figure 1

Note that we have not proved the uniqueness of $A$ and $B$.

Hence, we obtain a sequence of transformations with the invariant domain $\mathscr{R}$ between the invariant curve of type $\left(K_{1},+L_{1}\right)$ and its $S$-image (these two curves cut the torus $\Pi^{2}$ into two parts, $\mathscr{R}$ is the one containing the diagonal $x_{1}=x_{2}$ ). In this domain, we have the invariant polygon $\mathscr{P}$ bounded by the invariant curve of type $\left(K_{2},-L_{2}\right)$. We shall consider the invariant domain $\mathscr{D}=\mathscr{R} \backslash$ int $\mathscr{P}$. So the domain $\mathscr{D}$ and the corresponding transformation $T$ depend on four natural parameters $K_{1}$, $K_{2}, L_{1}, L_{2}$. For simplicity of notation we will not indicate this dependence explicitly.

There is the hyperbolic fixed point $(b, b)$ in $\mathscr{D}$. If the global unstable 'manifold' of the fixed point coincides with the global stable 'manifold', then they form an invariant curve of type $(K,+\infty)$ with $K_{1}<K<K_{2}$. Hence, if $K_{2}-K_{1}=1$, then the global stable and unstable manifolds intersect transversally on the axis of the symmetry $\boldsymbol{P}_{-a}$, i.e. there exists a homoclinic point in $\mathscr{D}$ (figure 2 ).

The domain $\mathscr{D}$ can thus be considered as a thin layer around intersecting stable and unstable manifolds of a fixed hyperbolic point, where there are no more invariant curves except for the curves contractible in the domain to a point (which encircle elliptic periodic points). This kind of domain can be considered for smooth perturbations of the twist map. There is a common belief that, in such domains, the stochastic behaviour is best developed (of course in general elliptic period points can be dense in them [3]). In the next paragraph, we prove that, under some assumptions for our piecewise linear transformation $T$, there are no elliptic periodic points in $\mathscr{D}$ and $T$ has strong mixing properties in it (is almost hyperbolic).

\section{Almost hyperbolicity of $T$ in $\mathscr{D}$}

For the sake of completeness, we repeat the definition of almost hyperbolicity from [2].

DEFINITION. We say that a piecewise linear measure-preserving transformation $T: \Pi^{2} \rightarrow \Pi^{2}$ is almost hyperbolic in an invariant domain $\mathscr{K} \subset \Pi^{2}$ if and only if there are two families of disjoint segments passing through almost all points of $\mathscr{K}$, called 


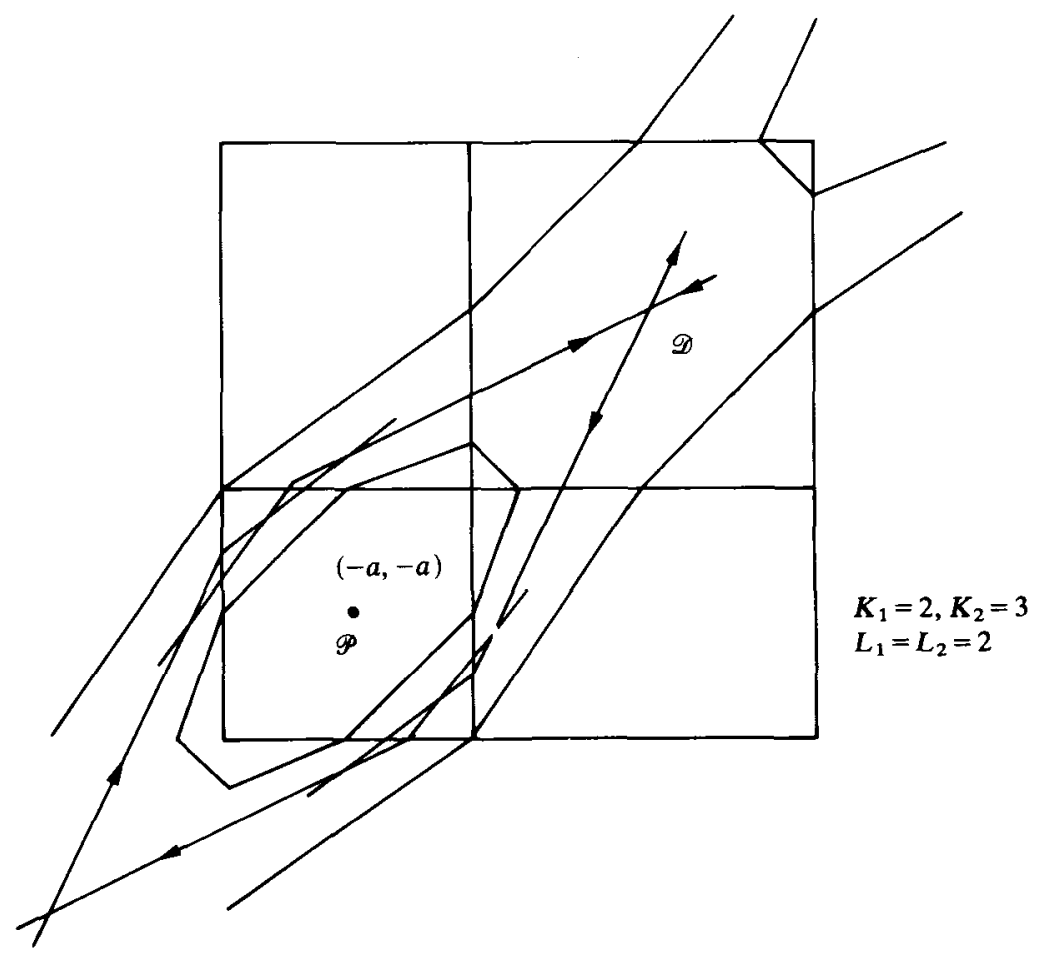

FIGURE 2

respectively the local contracting and expanding fibres, with the following properties. Denote by $\gamma^{s}(p),\left(\gamma^{u}(p)\right)$ the local contracting (expanding) fibre passing through $p \in \mathscr{K}$.

(i) $\gamma^{s}(p)$ and $\gamma^{u}(p)$ intersect transversally;

(ii) $T\left(\gamma^{s}(p)\right) \subset \gamma^{s}(T(p))$ and $T^{-1}\left(\gamma^{u}(p)\right) \subset \gamma^{u}\left(T^{-1}(p)\right)$;

(iii) if $p_{1}, p_{2} \in \gamma^{s}(p)\left(\gamma^{u}(p)\right)$, then, for $n>0(n<0)$,

$$
\operatorname{dist}\left(T^{n} p_{1}, T^{n} p_{2}\right)<\text { const } \lambda^{|n|} \text {, }
$$

where $0<\lambda<1$ and $\lambda$ and the constant depend on the fibre.

Actually almost hyperbolicity as defined above is equivalent to the non-vanishing of the Lyapunov characteristic exponents almost everywhere in $\mathscr{K}$. The consequence of almost hyperbolicity are the strong mixing properties of $T$ in $\mathscr{K}$. Namely, there is a family of invariant subsets of positive measure $\mathscr{K}_{i}, i=1,2, \ldots$,

$$
\bigcup_{i=1}^{\infty} \mathscr{K}_{i}=\mathscr{K}
$$

such that $\left.T\right|_{\mathscr{K}_{i}}$ is ergodic. Moreover,

$$
\begin{gathered}
\mathscr{K}_{i}=\mathscr{K}_{i}^{1} \cup \cdots \cup \mathscr{K}_{i}^{n_{i}}, \\
\mathscr{K}_{i}^{K} \cap \mathscr{K}_{i}^{L}=\varnothing, \quad \text { if } K \neq L, \\
T_{\mathscr{K}_{i}^{L}}^{L}=\mathscr{K}_{i}^{L+1}, \quad L=1, \ldots, n_{i}-1, \\
\operatorname{TK}_{i}^{n_{i}}=\mathscr{K}_{i}^{1},
\end{gathered}
$$


and $\left.T^{n_{i}}\right|_{\mathscr{K}_{i}^{1}}$ is Bernoulli (see [4] and references in it). The question of ergodicity (number of ergodic components) has to be decided individually.

To establish almost hyperbolicity for $S$-reversible transformations it suffices to find only expanding fibres. Then contracting fibres are given by

$$
\gamma^{s}(p)=S\left(\gamma^{u}(S p)\right) .
$$

We will use the following criterion of almost hyperbolicity for $S$-reversible transformations $T$.

Let $\mathscr{K}$ be some domain on the torus not necessarily invariant and suppose for almost all points $p \in \mathscr{K}$, there is a sector $U(p)$ in the tangent space so that the following properties hold:

(i) $D T_{\mathscr{K}}(U(p)) \subset U\left(T_{\mathscr{T}} p\right)$ where $T_{\mathscr{K}}: \mathscr{K} \rightarrow \mathscr{K}$ is the first return map;

(ii) for $v \in U(p),\left\|D T_{\mathscr{S}}\right\|\|\geq\| v \|$ and for almost all $p \in \mathscr{K}$, there are $\lambda, 0<\lambda<1$, and $L>0$ such that

$$
\left\|D T_{\mathscr{R}}^{L} v\right\| \geq \lambda^{-1}\|v\| \quad \text { for } v \in U(p) ;
$$

(iii) there is $\mu, 0<\mu \leq 1$, such that, for every $v \in U(p)$ and for every $n \geq 0$,

$$
\left\|D T^{n} v\right\| \geq \mu\|v\| .
$$

$\|\cdot\|$ denotes some smooth norm (defined, for example, by a Riemannian metric).

THEOREM 1. If an S-reversible transformation $T$ satisfies (i)-(iii), then it is almost hyperbolic in $\mathscr{K}_{1}$,

$$
\mathscr{K}_{1}=\bigcup_{i=-\infty}^{+\infty} T^{i} \mathscr{K} .
$$

This criterion was proved in [2] (theorem 2) for the special case of $\mu=1$. The proof of the proposed generalization is almost the same so it will be omitted. Actually, assumption (iii) could be dropped but instead of it, we would have to study the singularity lines of the first return map $T_{\mathscr{K}}$ (see [5]).

Applying theorem 1, we will obtain the following main result.

THEOREM 2. If $K_{2}-K_{1}=1$ and $L_{2} \leq L_{1} \leq L_{2}+1$, then $T$ is almost hyperbolic in $\mathscr{D}$.

Proof. It is convenient to go over to coordinates $\left(y_{1}, y_{2}\right)$ :

$$
y_{1}=x_{1}-\cos \alpha x_{2}, \quad y_{2}=\sin \alpha x_{2},
$$

in which $T$ (and $D T$ ) in $\mathscr{B}_{-}$is the rotation by the angle $-\alpha$.

Consider the domain $\mathscr{D}_{-}=\mathscr{D} \cap \mathscr{B}_{-} \cap \mathscr{C}_{+}$. It consists of two quadrilaterals. We take $\mathscr{D}_{-}$for $\mathscr{K}$ in theorem 1. (It is helpful to keep in mind that, in view of (3) the transformation $T$ factors by central symmetry so that the dynamics of both com-

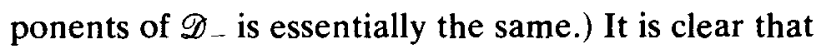

$$
\bigcup_{i=-\infty}^{+\infty} T^{i} \mathscr{D}
$$

coincides with $\mathscr{D}$ without the hyperbolic fixed point.

Consider the constant vector bundle $U$ on $\mathscr{D}_{\text {- }}$ defined in the following way. By the construction of the invariant curves, the unstable line of the hyperbolic fixed point cuts the quadrilaterals constituting $\mathscr{D}$. Take the opposite sides of one of the 
quadrilaterals which are not cut by the unstable line. Further take two lines in the tangent plane with the direction of these sides and put $U$ to be the sector between them containing the unstable line of $M_{+}$. The unstable line of $M_{+}$lies in $U$ but not the stable line so, under the application of $M_{+}, U$ goes into itself.

We will check assumptions (i)-(iii) of theorem 1 .

Assumption (i). $D T_{\mathscr{D}_{-}} U \subset U$. Points from $\mathscr{D}_{-}$leave $\mathscr{B}_{-}$either under $T^{K-1}$ or $T^{\mathrm{K}}$ where $K=K_{1}=K_{2}-1$. Denote the corresponding subsets of $\mathscr{D}_{-}$by $\mathscr{D}_{-}^{K-1}$ and $\mathscr{D}_{-}^{K}$ (obviously in one component of $\mathscr{D}_{-}$, they are triangles see figure 3 ).

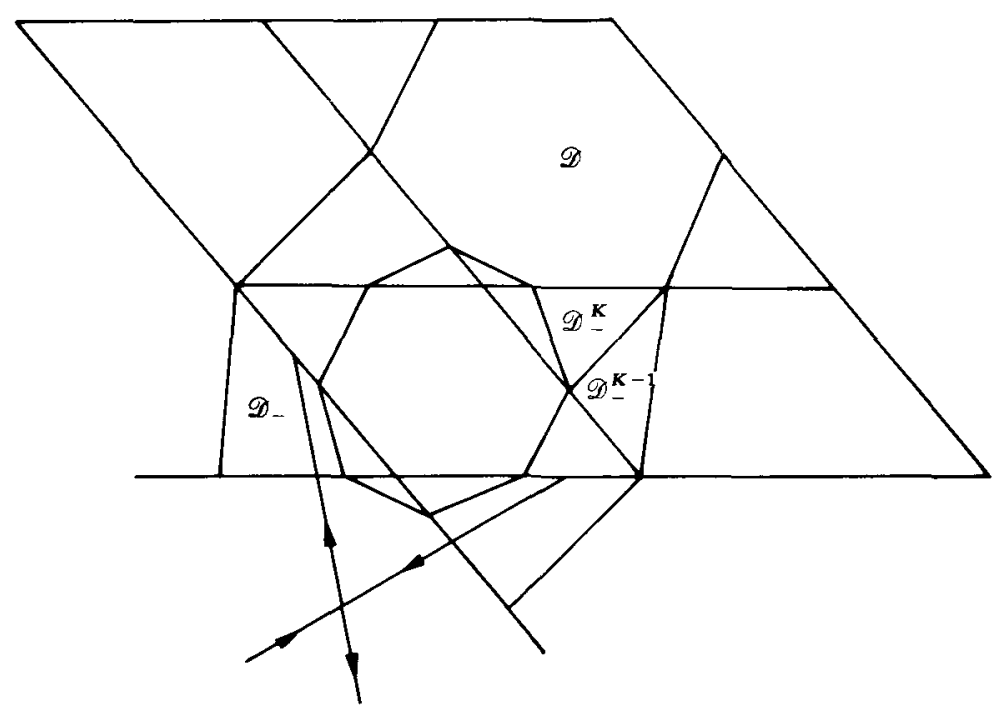

FIGURE 3

Consider the sector $P U$ which in particular contains the stable line of $M_{+}$. We use the same letter $P$ for the symmetry $P_{-a}=P_{b}=P$ on the torus and for its differential in the tangent plane. Because of the symmetry properties of the invariant curves, we obtain that, for $p \in \mathscr{D}_{-}^{K^{-1}}$,

$$
\left(D T^{K-1}\right)_{p} U=M_{-}^{K-1} U \underset{\text { def. }}{=} U_{K-1}
$$

is a sector which has a common line with $P U$ and lies outside of $P U$ (between $P U$ and $U$ with respect to counterclockwise orientation). Analogously, for $p \in \mathscr{D}_{-}^{K}$,

$$
\left(D T^{K}\right)_{p} U=M_{-}^{K} U \underset{\text { def. }}{=} U_{K}
$$

is a sector which has a common line with $P U$ and lies between $U$ and $P U$ (see figure 4).

After the point $p \in \mathscr{D}_{-}$has left $\mathscr{B}_{-}$, it can move either along the curve of the type $\left(K+1,-L_{2}\right)$ or of the type $\left(K,+L_{1}\right)$ (it depends on which side of the stable 'manifold' of the hyperbolic fixed point we are). Hence, before leaving $\mathscr{B}_{+}$, we apply $M_{+}$at least $L_{2}$ times in the first case and at least $L_{1}$ times in the second. By 


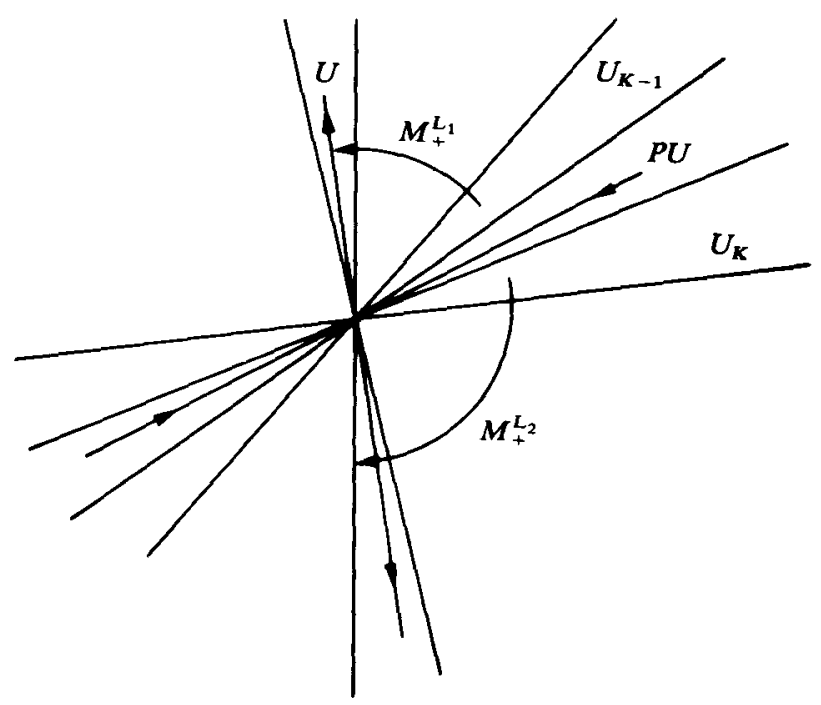

FIGURE 4

the symmetry of invariant curves, we obtain that the sector

$$
M_{+}^{L_{2}} U_{K} \underset{\text { def. }}{=} U_{K, L_{2}}
$$

is contained in $U$ and has a common side with it. Analogously, the sector

$$
M_{+}^{L_{1}} U_{K-1} \underset{\text { def. }}{=} U_{K-1, L_{1}}
$$

is contained in $U$ and has a common side with it (the other side of $U$ ). A point from $T^{K-1}\left(\mathscr{D}_{-}^{K-1}\right)=P \mathscr{D}_{-}^{K-1}$ leaves $\mathscr{B}_{+}$not earlier than under the iteration $T^{L_{1}}$ if it goes along the curve of type $\left(K,+L_{1}\right)$ and not earlier than under the iteration $T^{L_{2}+1}$ (!) if it goes along the curve of type $\left(K+1,-L_{2}\right)$. This conclusion can be obtained by the inspection of figure 3 . In the same manner, a point from $T^{K}\left(\mathscr{D}_{-}^{K}\right)=$ $P \mathscr{D}_{-}^{K}$ leaves $\mathscr{B}_{+}$not earlier than under the iteration $T^{L_{1}}$ if it goes along the curve of type $\left(K,+L_{1}\right)$ and not earlier than under the iteration $T^{L_{2}}$ if it goes along the curve of type $\left(K+1,-L_{2}\right)$.

We conclude that the necessary and sufficient condition for the preservation of the constant sector bundle $U$ by $D T_{\mathscr{O}_{-}}$is $L_{2}+1 \geq L_{1}$ and $L_{1} \geq L_{2}$. (i) is proved.

Assumption (ii). From the previous analysis, it follows that $M_{+}^{L_{1}} M_{-}^{K-1}$ is equal to the identity on the common line of the sectors $U$ and $U_{K-1, L_{1}}$. Hence $M_{+}^{L_{1}} M_{-}^{K-1}$ is a Jordan block type matrix and it is not difficult to see that, for $v \in U$,

$$
\left\|M_{+}^{L_{1}} M_{-}^{K-1} v\right\| \geq\|v\|
$$

where $\|\cdot\|$ denotes the Euclidean norm in the coordinates $\left(y_{1}, y_{2}\right)$.

Analogously $M_{+}^{L_{2}} M_{-}^{K}$ is equal to minus the identity on the common line of the sectors $U$ and $U_{K, L_{2}}$ and so it is also a Jordan block type matrix and for $v \in U$

$$
\left\|M_{+}^{L_{2}} M_{-}^{K} v\right\| \geq\|v\| \text {. }
$$


We conclude that, for $v \in U$,

$$
\left\|D T_{\mathscr{D}_{-}} v\right\| \geq\|v\|
$$

i.e. the differential of the first return map at least does not decrease the norm of vectors from $U$. Moreover it is not difficult to see that, if we stay in $\mathscr{B}_{+}$for a time longer than minimal, then the vectors from $U$ will be stretched under the return to $\mathscr{D}_{-}$. More exactly, there is $\lambda, 0<\lambda<1$, such that, for $v \in U$,

$$
\left\|M_{+}^{L} M_{-}^{K-1} v\right\| \geq \lambda^{-1}\|v\| \quad \text { if only } L \geq L_{1}+1
$$

and

$$
\left\|M_{+}^{L} M_{-}^{K} v\right\| \geq \lambda^{-1}\|v\| \quad \text { if only } L \geq L_{2}+1 .
$$

Moreover, for $v \in U$ and $\lambda$ sufficiently close to 1 ,

$$
\left\|\left(M_{+}^{L_{2}} M_{-}^{K}\right)\left(M_{+}^{L_{1}} M_{-}^{K-1}\right) v\right\| \geq \lambda^{-1}\|v\|
$$

and

$$
\left\|\left(M_{+}^{L_{1}} M_{-}^{K-1}\right)\left(M_{+}^{L_{2}} M_{-}^{K}\right) v\right\| \geq \lambda^{-1}\|v\| .
$$

So, if, for a point $p \in \mathscr{D}_{-}$, there is no $L \geq 1$ such that, for $v \in U$,

then, either

$$
\left\|D T_{\mathscr{D}_{-}}^{L} v\right\| \geq \lambda^{-1}\|v\|,
$$

or

$$
p \in \bigcap_{i=0}^{+\infty} T^{-i\left(L_{1}+K-1\right)} \mathscr{D}_{-}^{K-1}
$$

$$
p \in \bigcap_{i=0}^{+\infty} T^{-i\left(L_{2}+K\right)} \mathscr{D}_{-}^{K} .
$$

But it is not difficult to see that these sets have zero measure (they coincide with the boundary segments of $\mathscr{D}_{-}^{K-1}$ and $\mathscr{D}_{-}^{K}$ respectively).

Assumption (iii). When we apply $M_{+}$to a vector from sectors $U_{K-1}$ or $U_{K}$, then its norm decreases. But, since these sectors are bounded away from the stable line of $M_{+}$(they lie outide of $P U$ ), there is $\mu, 0<\mu<1$, such that, for all $n \geq 1$,

$$
\left\|M_{+}^{n} v\right\| \geq \mu\|v\| \quad \text { if } v \in U_{K-1} \cup U_{k} .
$$

Hence assumptions (i)-(iii) hold and our theorem follows from theorem 1.

In particular, if we take $K_{1}=2, K_{2}=3, L_{1}=1, L_{2}=2$, then we obtain $A=B=1$ and our theorem gives us theorem 6 of [2].

\section{Asymptotics of the area of $D$}

Denote by $A_{K, L}$ and $B_{K, L}$ the parameters for which the transformation $T$ has invariant curves of types $(K,+L)$ and $(K+1, L)$ (we have not proved that such parameters are unique for given $K, L$ but luckily, we shall not need it).

THEOREM 3. If $L=z K \ln K(1+o(1)), z>0$, then

where $2 \sqrt{t} \operatorname{arctg} \sqrt{t}=z^{-1}$.

$$
\lim _{K \rightarrow+\infty} \frac{B_{K, L}}{\boldsymbol{A}_{K, L}}=t
$$


In the proof, we shall need the following lemmas.

Let us denote by $\sigma$ the angle between the stable and unstable lines of $M_{+}$in the $y$-coordinates (introduced in the beginning of the proof of theorem 2).

LeMma 3.

$$
\operatorname{tg} \sigma / 2=\operatorname{th} \beta / 2 \operatorname{ctg} \alpha / 2 .
$$

Proof. In the $y$-coordinates, the stable $v_{s}$ and unstable $v_{u}$ eigenvectors of $M_{+}$have the form

$$
v_{s}=\left(e^{\beta}-\cos \alpha, \sin \alpha\right), \quad v_{u}=\left(1-\cos \alpha e^{\beta}, \sin \alpha e^{\beta}\right)
$$

(the eigenvalues of $M_{+}$are $e^{\beta}$ and $e^{-\beta}$ ).

So we obtain

$$
\begin{gathered}
\cos \sigma=\frac{-\cos \alpha e^{2 \beta}+2 e^{\beta}-\cos \alpha}{e^{2 \beta}-2 \cos \alpha e^{\beta}+1}, \quad \sin \alpha=\frac{\left(e^{2 \beta}-1\right) \sin \alpha}{e^{2 \beta}-2 \cos \alpha e^{\beta}+1}, \\
\operatorname{tg} \sigma / 2=\frac{1-\cos \sigma}{\sin \sigma}=\frac{(1+\cos \alpha)\left(e^{\beta}-1\right)^{2}}{\sin \alpha\left(e^{2 \beta}-1\right)} \\
=\operatorname{th} \beta / 2 \operatorname{ctg} \alpha / 2 .
\end{gathered}
$$

LEMMA 4.

$$
\operatorname{sh} L \beta=\sin \sigma \operatorname{ctg} \alpha / 2 .
$$

Proof. Subtracting (6) from (7), we get

$$
\operatorname{tg} K \alpha / 2-\operatorname{tg}(K-1) \alpha / 2=2 \operatorname{tg} \sigma / 2 / \operatorname{sh} L \beta .
$$

Multiplying (6) by (7), we obtain

Hence,

$$
\operatorname{tg} K \alpha / 2 \operatorname{tg}(K-1) \alpha / 2=\operatorname{tg}^{2} \sigma / 2 .
$$

$$
\operatorname{tg} \alpha / 2=\sin \sigma / \operatorname{sh} L \beta .
$$

Proof of theorem 3. For the simplicity of notation, we will drop the indices $K, L$ in $A_{K, L}, B_{K, L}$ and in corresponding to them $\alpha, \beta$ and $\sigma$.

Consider a subsequence $K_{s}$ such that

$$
\lim _{s \rightarrow+\infty} \sigma=\sigma_{0}, \quad 0 \leq \sigma_{0} \leq \pi .
$$

We will prove that, for such a subsequence,

$$
\lim _{s \rightarrow \infty} \beta / \alpha=\sqrt{t},
$$

proving thus that, for the whole sequence,

$$
\lim _{K \rightarrow+\infty} B / A=t .
$$

We have that $(K-1) \alpha<\sigma<K \alpha$ which follows immediately from lemma 3, (6) and (7) (or from figure 4). It follows that

$$
\lim _{s \rightarrow+\infty} K_{5} \alpha=\sigma_{0}
$$

and hence, in view of lemma 4 ,

$$
\lim _{s \rightarrow+\infty} \operatorname{sh} L \beta / K_{s}=2 \sin \sigma_{0} / \sigma_{0} .
$$


Since the last limit is always finite, then we must have

$$
\lim _{s \rightarrow+\infty} \beta=0 \text {. }
$$

In view of lemma 3 ,

$$
\lim _{s \rightarrow+\infty} \beta / \alpha=\operatorname{tg} \sigma_{0} / 2
$$

and hence

$$
\lim _{s \rightarrow+\infty} K_{s} \beta=\sigma_{0} \operatorname{tg} \sigma_{0} / 2
$$

From (8), we obtain

$$
\lim _{s \rightarrow+\infty} e^{L \beta} / K_{s}=\left(2 \sin \sigma_{0}\right) / \sigma_{0} .
$$

But, by the assumption of our theorem,

$$
e^{L \beta} / K_{\mathrm{s}}=K_{s}^{\left(z+\varepsilon\left(K_{s}\right)\right) K_{s} \beta^{-1}}
$$

where $\varepsilon(K)$ converges to 0 when $K \rightarrow+\infty$. So, we must have

$$
\lim _{s \rightarrow+\infty} K_{s} \beta=z^{-1} \text {. }
$$

We conclude that

$$
\lim _{s \rightarrow+\infty} \beta / \alpha=\sqrt{t}
$$

where

$$
t=\operatorname{tg}^{2} \sigma_{0} / 2 \text { and } \sigma_{0} \operatorname{tg} \sigma_{0} / 2=z^{-1}
$$

In particular, if

$$
L=(2 / \pi) K \ln K(1+o(1))
$$

then

$$
\lim _{K \rightarrow+\infty} B / A=1 \text { and } \lim _{K \rightarrow+\infty} \sigma=\pi / 2
$$

Hence asymptotically, $A=B$. In the following, we shall consider only this case and compute the asymptotics of the area of the domain $\mathscr{D}$ in which $T$ is almost hyperbolic.

Using the area-preserving property of $T$, we get immediately

$$
\text { Area }\left(\mathscr{D} \cap\left(\mathscr{B}_{-} \cup \mathscr{G}_{-}\right)\right)=(K+1) \text { Area }\left(\mathscr{D}_{-}^{K}\right)+K \text { Area }\left(\mathscr{D}_{-}^{K-1}\right) \text {. }
$$

Obviously (see figures 2 and 3 ),

$$
\begin{aligned}
\text { Area }\left(\mathscr{D}_{-}^{K}\right) & =\left(u_{K}-u_{K+1}\right)\left(u_{K+1}+c / 4\right), \\
\text { Area }\left(\mathscr{D}_{-}^{K-1}\right) & =\left(u_{K}-u_{K+1}\right) u_{K} .
\end{aligned}
$$

The area is computed in the $x$-coordinates.

After simple geometric considerations, we also obtain

$$
\text { Area }\left(\mathscr{D} \cap \mathscr{B}_{+} \cap \mathscr{G}_{+}\right)=(L-1) \text { Area }\left(\mathscr{D}_{-}\right)+2\left(u_{K}-u_{K+1}\right) b .
$$

THEOREM 4.

$$
\operatorname{Area}(\mathscr{D})=\frac{1}{16} A \ln \frac{1}{A}(1+o(1))
$$


Proof. In view of (4),

$$
\begin{aligned}
u_{K}-u_{K+1} & =\frac{c}{8} \frac{\cos \alpha / 2}{\sin (K-1) \alpha / 2 \sin K \alpha / 2}=\frac{A}{4}(1+o(1)), \\
u_{K}+u_{K+1}+\frac{c}{4} & =\frac{c}{8} \operatorname{ctg} \alpha / 2 \frac{\sin (2 K-1) \alpha / 2}{\sin (K-1) \alpha / 2 \sin K \alpha / 2}+\frac{c}{4} \\
& =\frac{\sqrt{A}}{2}(1+o(1)) .
\end{aligned}
$$

It is not difficult to see that the main contribution to the asymptotics of the area of $\mathscr{D}$ as $A \rightarrow 0$ is $L$ Area (D_-). But,

$$
K=\pi / 2 \sqrt{A}(1+o(1))
$$

so that

$$
L=(1 / \sqrt{A}) \ln (1 / \sqrt{A})(1+o(1))
$$

Finally, we obtain

$$
L \operatorname{Area}\left(\mathscr{D}_{-}\right)=\frac{1}{16} \ln \frac{1}{A}(1+o(1)) .
$$

We know that the stable and unstable separatrices of the hyperbolic fixed point $(b, b)$ intersect transversally. It is interesting to see what is the asymptotics of the angle of intersection. We take the point of intersection on the axis of symmetry $P_{-a}$. Denote the angle of intersection in $x$-coordinates by $x$.

PROPOSITION 2.

$$
\chi=A(1+o(1)) \text {. }
$$

Proof. We shall first consider the angle of intersection in $y$-coordinates. Denote it by $\xi$. It is clear (see figures 2 and 4 ) that

$$
\xi=\sigma-(K-1) \alpha
$$

if $K$ is odd and

$$
\xi=K \alpha-\sigma
$$

if $K$ is even. But we have

$$
\sigma-(K-1) \alpha=\frac{\alpha}{2}(1+o(1)) \quad \text { and } \quad K \alpha-\sigma=\frac{\alpha}{2}(1+o(1)) .
$$

Indeed,

$$
\frac{\operatorname{tg}\left(\frac{\sigma}{2}-(K-1) \frac{\alpha}{2}\right)}{\operatorname{tg}\left(K \frac{\alpha}{2}-\frac{\sigma}{2}\right)}=\frac{\left(1+\operatorname{tg} K \frac{\alpha}{2} \operatorname{tg} \frac{\sigma}{2}\right)}{\left(1+\operatorname{tg}(K-1) \frac{\alpha}{2} \operatorname{tg} \frac{\sigma}{2}\right)} \frac{\left(\operatorname{tg} \frac{\sigma}{2}-\operatorname{tg}(K-1) \frac{\alpha}{2}\right)}{\left(\operatorname{tg} K \frac{\alpha}{2}-\operatorname{tg} \frac{\sigma}{2}\right)}
$$

By (6), (7) and lemma 3,

$$
\begin{aligned}
\operatorname{tg}(K-1) \alpha / 2 & =\operatorname{th} L \beta / 2 \operatorname{tg} \sigma / 2, \\
\operatorname{tg} K \alpha / 2 & =\operatorname{cth} L \beta / 2 \operatorname{tg} \sigma / 2 .
\end{aligned}
$$


Hence

$$
\begin{aligned}
\frac{\operatorname{tg}(\sigma / 2-(K-1) \alpha / 2)}{\operatorname{tg}(K \alpha / 2-\sigma / 2)} & =\frac{1-\operatorname{th} L \beta / 2}{\operatorname{cth} L \beta / 2-1}(1+o(1)) \\
& =\operatorname{th} L \beta / 2(1+o(1))=o(1)
\end{aligned}
$$

Thus we have

$$
\xi=\frac{\alpha}{2}(1+o(1))
$$

The stable and unstable separatrices at the point of intersection have, in $y$ coordinates, directions of the vectors:

$$
(\sin (\alpha / 2+\xi / 2), \cos (\alpha / 2+\xi / 2)) \text { and }(\sin (\alpha / 2-\xi / 2), \cos (\alpha / 2-\xi / 2)) \text {. }
$$

Returning to the $x$-coordinates, we obtain that the directions are now given by the vectors

$$
(\cos (\alpha / 2-\xi / 2), \cos (\alpha / 2+\xi / 2)) \text { and }(\cos (\alpha / 2+\xi / 2), \cos (\alpha / 2-\xi / 2)) .
$$

So we have

Hence,

$$
\begin{aligned}
\sin \chi & =\frac{\cos ^{2}(\alpha / 2-\xi / 2)-\cos ^{2}(\alpha / 2+\xi / 2)}{\cos ^{2}(\alpha / 2-\xi / 2)+\cos ^{2}(\alpha / 2+\xi / 2)} \\
& =2 \sin \alpha \sin \xi(1+o(1)) .
\end{aligned}
$$

$$
\chi=\alpha^{2}(1+o(1))
$$

\section{Appendix}

The complexity of the stucture of ergodic components of positive measure of a smooth perturbation of the twist map (if there is any at all) stems from the presence of an infinite number of elliptic periodic points which are generically dense [3]. We found domains with almost hyperbolicity for the piecewise linear perturbations and hence with no elliptic periodic points in them. A question arises: can the transformation $T$ have infinitely many elliptic periodic points with different periods? In this appendix, we prove the following theorem.

THEOREM 5. For parameters $A$ and $B$ for which $T$ has the invariant curve of type $(K, \infty), K \geq 2$ (i.e. $\left.u_{K}=v_{\infty}\right)$, there is an infinite number of elliptic periodic points with different periods.

In particular, for $A=B$, there is a sequence of parameters $A_{K} \searrow 0, K \geq 2$, for which the stable and unstable 'manifolds' of the hyperbolic fixed point coincide (the invariant curve of the type $(K, \infty)$ ). For these parameters, the transformation $T$ has an infinite set of elliptic periodic points with different periods.

Proof. Let us consider the domain bounded by the coinciding stable and unstable 'manifolds' (the separatrix) of the hyperbolic fixed point $(b, b)$ and containing the elliptic fixed point $(-a,-a)$. Consider the segment $\zeta$ with ends

$$
p_{K}=\left(u_{K}, 0\right)=\left(v_{\infty}, 0\right) \text { and } T p_{K}=\left(0,-u_{K}-c / 4\right) \text {, }
$$

contained in the separatrix. This segment has the direction of the unstable line of the hyperbolic fixed point $(b, b)$. 
Denote the centre of $\zeta$ by $s$. We have

$$
T^{K-1} s=P_{-a} s \text { and } T^{i} s \in \mathscr{B}_{-} \quad \text { for } 0 \leq i \leq K-2 .
$$

All the points from the segment $\eta$ with ends $(-a,-a)$ and $s$ have the same property, i.e., for $p \in \eta$,

$$
T^{K-1} p=P_{-a} p \quad \text { and } \quad T^{i} p \in \mathscr{B}_{-} \text {for } 0 \leq i \leq K-2 .
$$

At the same time, there is a sequence of points $q_{L} \in \eta, L=n, n+1, \ldots, q_{L} \rightarrow s$ as $L \rightarrow+\infty$, where $n$ is sufficiently large, such that

$$
T^{-L} q_{L}=S q_{L} \text { and } T^{-i} q_{L} \in \mathscr{G}_{+} \text {for } 0 \leq i \leq L-1 .
$$

We obtain these points as the intersection of $\eta$ and $T^{L} S \eta$.

By the symmetry properties of $T\left((1),(2)\right.$ and (3)), all $q_{L}, L \geq n$, are periodic points with periods $2(L+K-1)$. The differential of $T^{2(L+K-1)}$ at $q_{L}$ clearly has the form $\left(M_{+}^{L} M_{-}^{K-1}\right)^{2}$.

We claim that all $q_{L}$ are elliptic periodic points. It suffices to prove that the matrix $M_{+}^{L} M_{-}^{K-1}$ is elliptic. But clearly $M_{-}^{K-1}$ takes the unstable line of $M_{+}$(of the hyperbolic fixed point) onto the stable line. So in the basis of eigenvectors of $M_{+}$,

$$
M_{+}=\left(\begin{array}{cc}
e^{\beta} & 0 \\
0 & e^{-\beta}
\end{array}\right) \text { and } M_{-}^{K-1}=\left(\begin{array}{cc}
0 & -1 \\
1 & d
\end{array}\right)
$$

with $d \in(-2,2)$. The trace of $M_{+}^{L} M_{-}^{K-1}$ is thus equal to $e^{-L \beta} d$. Hence all the matrices $M_{+}^{L} M_{-}^{K-1}$ are elliptic. We conclude that $q_{L}, L=n, \ldots$, are elliptic periodic points with different periods.

Analogously, there is a sequence of elliptic periodic points converging to the separatrix outside of our domain.

This paper was written while the author was on leave from Warsaw University on a visit to the Department of Mathematics of the Facultés Universitaires Notre-Dame de la Paix à Namur, Belgium. The author is grateful for its hospitality and for the support of the Fonds National de la Recherche Scientifique, Belgique which made this visit possible. I thank Mrs Martine Frippiat-Van Caenegem for the excellent typing of the manuscript.

\section{REFERENCES}

[1] B. V. Chirikov. A universal instability of many dimensional oscillator systems. Physics Rep. 52 (1979), 263-379.

[2] M. Wojtkowski. A model problem with the coexistence of stochastic and integrable behaviour. Commun. Math. Phys. 80 (1981), 453-464.

[3] S. E. Newhouse. Quasi-elliptic periodic points in conservative dynamical systems. Amer. J. Math. 99 (1977), 1061-1087.

[4] Ya. B. Pesin. Lyapunov characteristic exponents and smooth ergodic theory. Usp. Mat. Nauk 32 No. 4 (1977), 55-112. (In Russian.) English translation in Russ. Math. Survey 32 No. 4 (1977), 55-114.

[5] A. Katok \& J.-M. Strelcyn. Invariant manifolds for smooth maps with singularities. Part I: Existence. Preprint (1980). 